\title{
Unethical Behaviour Witnessed by Medical Students During Their Medical Studies
}

\author{
Susy Kovatz* and Louis Shenkman
}

\author{
Department of Medicine "C", Meir Medical Center, Kfar Saba, Israel, and, The New York State/American Program, \\ Sackler School of Medicine, Tel Aviv University, Tel Aviv, Israel
}

\begin{abstract}
Background: We sought to determine students' perception of ethical behaviour of their peers and instructors during their course of studies, both in the preclinical and clinical years. To this end, we administered a questionnaire to senior medical students in order to assess whether they witnessed unethical behaviour during their four-year course of studies.
\end{abstract}

\begin{abstract}
Methods: A two-part questionnaire was distributed to all members of the graduating class of 2007. The first part of the questionnaire asked whether students ever witnessed unethical behaviour; whether it occurred during the preclinical or clinical years of study; whether the unethical behaviour witnessed was performed by a student, resident, senior physician, nurse or other; whether the student reported the unethical behaviour to anyone; and finally, if they discussed the event with their classmates. The second part of the questionnaire was based on one designed and utilized by [3].
\end{abstract}

Results: Forty of the 56 students responded to the questionnaire. Twenty-one students (52.5\%) responded that they witnessed unethical behaviour during their course of studies.

Three $(14.3 \%)$ observed the behaviour during their preclinical years, $10(47 \%)$ in their clinical years of study and 8 (31.8\%) during both the preclinical and clinical years. Thus, 11/21 (52.3\%) students reported witnessing unethical behaviour during their preclinical training and 18/21 students $(85.7 \%)$ during their clinical training. The eleven students who witnessed unethical behaviour during their preclinical training reported that six episodes $(52.5 \%)$ were by students and five $(45.4 \%)$ by faculty.

The eighteen students who reported unethical behaviour during their clinical years reported 2 episodes by students (11\%), 4 by residents (22\%) and 12 by faculty (67\%). The most common unethical behaviour observed was treating patients differently because of their background/race/beliefs ( $44.4 \%$ of episodes), followed by describing patients or their families in a derogatory manner and discussing confidential information in an inappropriate setting (38.8\% for both).

Conclusions: It is obvious from these findings that many students have the perception that they are witnessing unethical behaviour during their studies. Medical educators must move beyond the formal curriculum in order to reconstruct the overall learning environment of medical education so that students will be exposed to professional behaviour throughout their studies.

\section{BACKGROUND}

As practitioners of medicine, physicians are exposed to ethical issues and challenges on an almost daily basis. Most medical schools include in their curriculum formal studies on ethics, ethical behaviour and professionalism. However, the educational milieu of a medical student is determined by both the formal and the informal curriculum. In fact, Hafarty [1] identified three aspects that determine what is taught in medical school: the formally endorsed curriculum, the informal teaching and learning that takes place between faculty and students, and the "hidden" curriculum that a student absorbs from the organizational structure and culture of the medical environment. The hidden curriculum may play an important role in moulding student's professional identities

*Address correspondence to this author at the Department of Medicine "C", Meir Medical Center, Kfar Saba, Israel, and, The New York State/American Program, Sackler School of Medicine, Tel Aviv University, Tel Aviv, Israel; E-mails: susyk@clalit.org.il and in shaping their sense of values. The important influence of proper role modelling in the shaping of students' sense of values has been recognized as an essential element in effective medical education [2].

Because of the importance of "hidden" curriculum, we sought to determine students' perception of ethical behaviour of their peers and instructors during their course of studies, both in the preclinical and clinical years. To this end, we administered a questionnaire to senior medical students in order to assess whether they witnessed unethical behaviour during their four-year course of studies. This paper describes the results of the questionnaire and discusses its implications.

\section{METHODS}

A two-part questionnaire was distributed to all members of the graduating class of 2004. Their participation was voluntary and their responses anonymous. Other than asking students to participate in this survey, no other instructions were given. Specifically, they received no definition of what 
constitutes unethical behaviour prior to the questionnaire. The first part of the questionnaire asked whether students ever witnessed unethical behaviour; whether it occurred during the preclinical or clinical years of study; whether the unethical behaviour witnessed was performed by a student, resident, senior physician, nurse or other; whether the student reported the unethical behaviour to anyone; and finally, if they discussed the event with their classmates. The second part of the questionnaire was based on one designed and utilized by [3]. Students were asked to indicate whether they witnessed any of the following behaviours by physicians during their clinical rotations and electives: describing patients or their families in a derogatory manner; withholding or giving false information to patients or families; discussing confidential information in an inappropriate setting; treating patients differently because of their background/race/beliefs, etc.; treating non-physician health care workers in a disrespectful manner; describing other clinical services or other doctors in a derogatory manner. Finally, demographic data and background was collected, including the student's sex, whether they had any formal background in ethics in college, whether ethical issues were discussed in their home and if they believed that ethics received sufficient attention in their curriculum. All data were analyzed using SPSS software, version 11.0. Frequencies and percentage of all variables were computed. Each new variable was calculated to count the number of unethical behaviours witnessed by the students. To correlate background data with the witnessing of unethical behaviour, crosstabs and the Chi-Square tests were used. This study was approved by the university ethics committee.

\section{RESULTS}

Forty of the 56 students responded to the questionnaire. Thirteen $(33 \%)$ of the responders were women and $27(67 \%)$ were men. Twenty-one students $(52.5 \%)$ responded that they witnessed unethical behaviour during their course of studies.

Of the 21 students that witnessed unethical behaviour, 3 $(14.3 \%)$ observed the behaviour during their preclinical years, $10(47 \%)$ in their clinical years of study and $8(31.8 \%)$ during both the preclinical and clinical years. Thus, 11/21 $(52.3 \%)$ students reported witnessing unethical behaviour during their preclinical training and 18/21 students $(85.7 \%)$ during their clinical training.

The eleven students who witnessed unethical behaviour during their preclinical training reported that six episodes $(52.5 \%)$ were by students and five $(45.4 \%)$ by faculty.
The eighteen students who reported unethical behaviour during their clinical years reported 2 episodes by students $(11 \%), 4$ by residents $(22 \%)$ and 12 by faculty (67\%).

Six students $(29 \%)$ reported their experience to a member of the faculty or administration while $15(71 \%)$ did not. Ten students $(48 \%)$ discussed their observations with their classmates. The responses of the students to the various types of unethical behaviour observed during their clinical training are listed in Table $\mathbf{1 .}$

There was no statistically significant difference between men and women in the reporting of unethical behaviour: $39 \%$ women, $59 \%$ men $(\mathrm{P}=0.19)$. Having a college background in ethics did not affect the reporting of unethical behaviour nor did the discussion of ethics at home. A statistically significant difference in the reporting of unethical behaviour was noted between those students who believed that ethics received sufficient attention in the curriculum (33\%) and those who did not $(74 \%), \mathrm{P}=0.012$.

\section{DISCUSSION}

We have documented that over $50 \%$ of a medical school graduating class has witnessed behaviour that they interpret as unethical during their four years of study.

Of the students who reported observing unethical behaviour, $52 \%$ noted such behaviour during their preclinical years and $85.7 \%$ during their clinical training. Unethical behaviour in the clinical years was attributed in the majority to senior physicians ( $67 \%$ of episodes), to residents in $22 \%$ of reports and to fellow classmates for the remainder $(11 \%)$. In the breakdown of the types of unethical behaviour observed in the hospital (Table 1), the most common was treating patients differently because of their background/race/beliefs (44.4\% of episodes), followed by describing patients or their families in a derogatory manner and discussing confidential information in an inappropriate setting (38.8\% for both). Describing other clinical services or other doctors in a derogatory manner accounted for $33.3 \%$ of the reports, while withholding or giving false information to patients and families, and treating non-physician health care workers in a disrespectful manner were observed in $22 \%$ and $11.1 \%$ of the cases, respectively. Of interest, Szauter et al reported that the most common transgressions of faculty were derogatory comments directed at other services, at patients, or towards a patient's family [3].

A troubling finding was that while $48 \%$ of the students who observed unethical behaviour discussed their experi-

Table 1. Types of Unethical Behaviour Observed by Students During Their Clinical Rotations

\begin{tabular}{|c|c|}
\hline Type of Unethical Behaviour Observed & Number of Students \\
\hline \hline Describing patients or their families in a derogatory manner & $7(38.8 \%)$ \\
\hline Withholding or giving false information to patients or families & $4(22.2 \%)$ \\
\hline Discussing confidential information in an inappropriate setting & $7(38.8 \%)$ \\
\hline Treating patients differently because of their background/race/beliefs, etc. & $8(44.4 \%)$ \\
\hline Treating non-physician health care workers in a disrespectful manner & $2(11.1 \%)$ \\
\hline Describing other clinical services or other doctors in a derogatory manner & $6(33.3 \%)$ \\
\hline
\end{tabular}


ences with fellow classmates, only 6 students (29\%) reported their observations to either faculty or administration. This observation may suggest that reporting unethical behaviour to supervisors is an issue that should receive more attention in the curriculum. The correlation between witnessing unethical behaviour and background data of students deserves some comment. There was no statistically significant difference between men and women, nor was there a difference between students who had a background in ethics and those that did not. Of interest, $74 \%$ of students who believed that ethics did not receive sufficient attention in the formal curriculum reported witnessing unethical behaviour, compared to only $33 \%$ of those who felt ethics received sufficient attention. Assuming that all students were exposed to the same academic environment, this finding would support the subjective nature of the perception of unethical behaviour in students who felt that ethics requires greater emphasis in the curriculum, which is, of course, the major limitation of our study.

\section{CONCLUSIONS}

It is obvious from these findings that many students have the perception that they are witnessing unethical behaviour during their studies. Clearly, it is not sufficient to lecture about professionalism in the formal curriculum. Students must be witness to professionalism in their daily activities in the classrooms, in the hospital wards and in the clinics, and they must be exposed to role models who inevitably will mould their future behaviour as physicians. Physicians who undertake the responsibility as clinical teachers must be reminded of this important role that they fulfil. As Hafferty has so eloquently stated, medical educators must move beyond the formal curriculum in order to reconstruct the overall learning environment of medical education so that students will be exposed to professional behaviour throughout their studies [4].

\section{REFERENCES}

[1] Hafferty FW, Franks R. The hidden curriculum, ethics teaching, and the structure of medical education. Acad Med 1994; 69: 86171.

[2] Maudsley RF. Role models and the learning environment: essential elements in effective medical education. Acad Med 2001; 76: 4324

[3] Szauter K, Williams B, Ainsworth MA, Callaway M, Bulik R, Camp MG. Student perception of the professional behaviour of family physicians. Available from: http://www.med-ed-online.org Med Educ Online 2003; 8: 17

[4] Hafferty FW. Beyond curriculum reform: confronting medicine's hidden curriculum. Acad Med 1998; 73: 403-7. 